
\title{
25 Research Suare \\ Effects of the Hallux Valgus Angle on One-Leg Stance and Gait Parameters in Young Adults: A Preliminary Study
}

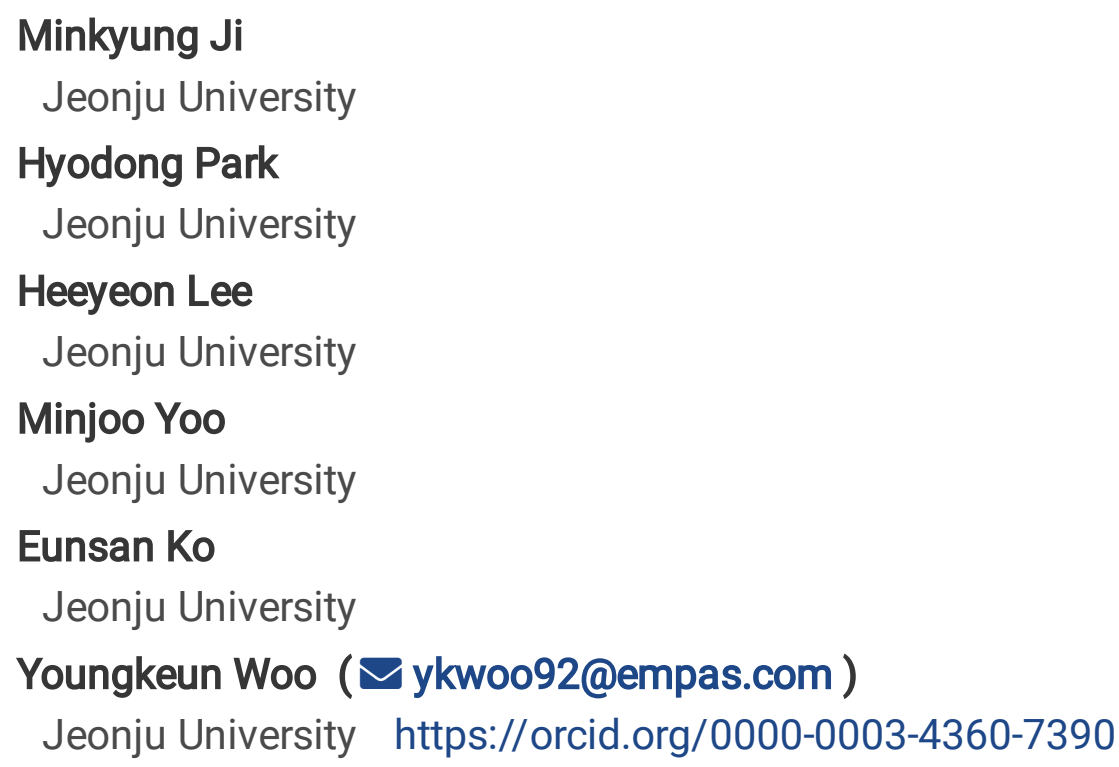

Research article

Keywords: Gait; Hallux valgus, One-leg stance

Posted Date: March 10th, 2020

DOI: https://doi.org/10.21203/rs.3.rs-16683/v1

License: () (1) This work is licensed under a Creative Commons Attribution 4.0 International License. Read Full License 


\section{Abstract}

Background Hallux valgus (HV) is the most common musculoskeletal deformity of the foot. This deformity is accompanied with pain and continues to decrease one's quality of life. Furthermore, HV has been reported to affect gait ability and static stability. This study aimed to compare one-leg stance and gait between people with and without $\mathrm{HV}$, and to investigate the effect of the HV angle on one-leg stance and gait ability. Methods Forty young adults with HV (6 men, 34 women) were enrolled. In the HV and normal groups, people who had undergone surgery or were unable to participate in the study because of pain in the lower extremity were excluded. Using the Zebris FDM model 1.5 (Zebris Medical GmbH, Isny, Germany), we analyzed gait parameters and one-leg stance in all subject to compare gait and static balance between the normal and HV groups. The obtained data were analyzed using the Mann-Whitney U-test to determine differences in one-leg stance and gait parameters between the group groups. In addition, a simple regression analysis was performed to determine the effect of the HV angle on one-leg stance and gait parameters. The significance level was set at $₫=0.05$. Results Significant differences were found in sway length and time change from the heel to forefoot during walking between the normal and HV groups. Most parameters were not associated with the HV angle, but a few one-leg stance and gait parameters were significantly associated with the HV angle. Conclusions These results suggest that most one-leg stance and gait parameters were not significantly affected by the HV angle in young adults; however, a few parameters were significantly different between the normal and HV groups. Because walking is a repetitive movement, young adults with $\mathrm{HV}$ could potentially have cumulative damage of the feet in later stages of life.

\section{Background}

Hallux valgus (HV) is one of the most common foot deformities requiring orthopedic care [1]. It is characterized as a lateral deformity of the extensor and flexor tendons of the big toe with a medial deviation of the first metatarsal head and a lateral deviation of the proximal phalanx of hallux, which occurs because the medial collateral ligament and joint capsule become attenuated [2]. The reported prevalences of HV were $28.4 \%$ in young adults [3] and as high as $74 \%$ in older adults [4]. HV is more common in female and elderly individuals, and the latter group has an increased risk for falls [5].

In general, $\mathrm{HV}$ is diagnosed by measuring anatomical angles: the angle between the first metatarsal and the hallux, the angle between the first and second metatarsals, and the distal metatarsal articular angle $[6,7]$. In radiographic measurements, a normal $H V$ angle (HVA) is $\leq 15^{\circ}$, and the first to second intermetatarsal angle is $\leq 8^{\circ}-9^{\circ}$ [8]. Pique-Vidal and Vila [9] classified HV deformities into normal $\left(<15^{\circ}\right)$, mild $\left(15^{\circ}-20^{\circ}\right)$, moderate $\left(21^{\circ}-39^{\circ}\right)$, and severe $\left(\geq 40^{\circ}\right)$ based on the HVA.

$\mathrm{HV}$ is characterized by pain, impaired balance, deformities of the lower limbs, and movement disorder related to lower limb kinetics, leading to a reduction of physical activity [10]. In addition, HV is distinguished by a greater reduction in the size of the abductor hallucis compared with the normal hallux [10] and/or by the collapse of the arch [2]. Suzuki et al. [11] claimed that HV can be accompanied with 
pain in the metatarsal and medial metatarsal arch collapse in the static position. Patients with $\mathrm{HV}$ have to bear loads caused by different lower extremity alignment compared with healthy individuals, which affects their walking ability [12]. Deschamps et al. [1] reported a significant difference in heel abduction angles during walking between HV and normal groups and that such a change can affect postural stability and balance. Wen et al. [13] reported a decreased single-leg stance performance in young adults who had HV that caused no pain and stated that HVA can potentially cause injury by deteriorating their single-leg stance performance during sports activities. According to Nishimura et al. [14], HV deformities with an HVA greater than $30^{\circ}$ decrease one's maximum walking speed, and painful HV negatively affects general gait features, including maximum walking speed. Plantar pressure is also decreased to varying degrees, depending on the severity of HVA, and it is significantly lower in patients with painful HA.

The first metatarsal joint acts as a pivot for transferring weight during the latter part of the stance phase of gait [15], and the force generation capacity of the plantar flexors in the first metatarsal is reduced because of the lateral deviation of the big toe $[2,16]$. Martinez-Nova et al. [17] reported no significant difference in the peak pressure between HV and control groups, whereas Bryant et al. [18] and Mickle et al. [19] asserted that the peak pressure increased, depending on the first and second metatarsal heads in patients with severe HA compared with the control group.

To describe foot pain and movements in relation to HA, dynamic measurements and assessments can be more reliable than their static counterparts [20]. Hence, in addition to the importance of HVA, the requirement of steady movement at different angles is seen as an essential component of evaluation when assessing patients with HA. This claim is supported by the fact that the ability to walk, once it is acquired as a baby, is likely to be maintained throughout life, and it is difficult to change gait characteristics naturally once the gait pattern has been fixed and repeated from the early stages of life [21].

The present study aimed to compare one-leg stance and gait between people with and without $\mathrm{HV}$, and to investigate the effect of the HV angle on one-leg stance and gait ability.

\section{Materials And Methods}

\section{Study subjects}

This cross-sectional study included 40 young adults ( 6 men and 34 women) with HV who had no pain in their lower extremities, including the hip and knee, and received no surgical treatment or physical therapy interventions to the lower extremities. Their weight, height, and HVAs from the left and right feet were measured, and demographic data such as age were collected. All subjects were right-foot dominant. During the experiments, those who complained of lower limb pain and those who did not want to disclose their measurements were excluded from the analysis.

The present study was approved by a local institutional review board (approval number: jjIRB-180713-HR2018-0708), and written informed consent form was obtained from each participant. 


\section{Measurement instruments and methods}

\section{1) HVA measurement with a goniometer}

The goniometer $(36 \times 4.5 \mathrm{~cm})$ has an axis in the center to facilitate $360^{\circ}$ rotation, and it was used to measure the HVA. While subjects were standing in their normal stance, the axis of the goniometer was placed on the first metatarsal joint, with the stationary arm being aligned with the first metatarsal and the moving arm being aligned with the great toe to measure HVA in accordance with the guidelines published by the Ad Hoc Committee of the American Orthopedic Foot \& Ankle Society [22].

2) Evaluation of unipedal balance and gait performance

The subjects' balance abilities in one-leg stance and gait performance were measured with the ZebrisFDM 1.5 (Zebris Medical GmbH, Isny, Germany). With dimensions of $580 \times 605 \times 21 \mathrm{~mm}$ (length $\times$ width $\times$ height), this pressure distribution measurement system is used to measure balance and gait parameters with 11,264 force sensors, which record data at a sampling rate of $120 \mathrm{~Hz}$ per 1 second. To measure oneleg stance balance, the subjects were asked to alternate standing on their right and left legs for 30 seconds each and rest for 1 minute. All measurements were repeated three times, and the average of the three scores was used in the analysis. In one-leg stance, subjects were asked to cross the lifted foot behind the back of the other foot and place it on the crook of the standing knee with their arms placed on their hips. While tests were conducted bilaterally, the order of the test leg was determined via a coin toss. For balance ability, the center of pressure (COP) path, velocity, length of axis of the COP path, deviation of the $x$-axis and $y$-axis, and percentage of foot pressure were measured.

For gait measurements, two units of the Zebris-FDM 1.5 were combined to make a 3-m-long measuring plate to which an additional $1.5-\mathrm{m}$ track was connected to make the start and end points. Subjects performed a 6-m walk test. At each measurement, the subjects were asked to look straight ahead to avoid potential visual biases. The test was repeated three times, and the average of the three scores was used in the analysis. For gait ability, the foot rotation angle, step length, percentage of each phase of the gait cycle, time change from the heel to forefoot, and maximum pressure of the forefoot and midfoot were measured.

\section{Statistical analysis}

Statistical analysis was performed using SPSS 25.0 software (IBM Corp., Armonk, NY, USA). All subjects were divided into the HV and normal groups, and an independent t-test was used to compare the differences in age, height, weight, and HVA between the groups. The non-parametric Mann-Whitney U-test was used to determine the differences in balance and gait parameters between the groups. To determine the relationship of HVA with parameters related to one-leg stance balance and gait performance, a simple regression analysis was performed using HVA as an independent variable and balance and gait parameters as dependent variables. Statistically significance level a was set to 0.05 . 


\section{Results}

\section{General characteristics}

General characteristics of participants are shown in Table 1.

Table 1

General characteristics of participants

\begin{tabular}{|c|c|c|c|c|c|}
\hline Side of foot & Parameters & $\begin{array}{l}\text { Normal adults } \\
(n=20)\end{array}$ & $\begin{array}{l}\text { HVa Adults } \\
(n=20)\end{array}$ & $t$ & $\mathbf{p}$ \\
\hline \multirow[t]{4}{*}{ Left } & Age (years) & $21.85 \pm 1.18^{b}$ & $20.70 \pm 1.17$ & 3.807 & 0.004 \\
\hline & Height (cm) & $164.29 \pm 7.86$ & $165.17 \pm 8.72$ & -0.335 & 0.739 \\
\hline & Weight (kg) & $58.10 \pm 10.23$ & $62.05 \pm 9.83$ & -1.245 & 0.221 \\
\hline & Angle of $\mathrm{HV}\left({ }^{\circ}\right)$ & $10.95 \pm 2.72$ & $23.85 \pm 5.05$ & -10.055 & 0.000 \\
\hline \multirow[t]{4}{*}{ Right } & Age (years) & $21.80 \pm 1.24$ & $20.75 \pm 1.16$ & 2.761 & 0.009 \\
\hline & Height $(\mathrm{cm})$ & $164.64 \pm 7.75$ & $164.82 \pm 8.84$ & -0.068 & 0.946 \\
\hline & Weight (kg) & $58.05 \pm 10.21$ & $62.10 \pm 9.83$ & -1.278 & 0.209 \\
\hline & Angle of $\mathrm{HV}\left({ }^{\circ}\right)$ & $10.30 \pm 3.67$ & $22.00 \pm 4.96$ & -8.475 & 0.000 \\
\hline
\end{tabular}

\section{Unipedal Balance Test On The Left Foot}

The results of the unipedal balance test on the left foot in the HV and normal groups are presented in Table 2. The length of the major and minor axes significantly increased in the HV group compared with the normal group $(p<0.05)$. The force $(\%)$ on the forefoot was significantly lower in the HV group than in the normal group $(p<0.05)$, whereas that on the back foot was significantly higher in the HV group than in the normal group $(p<0.05)$. However, there was no significant difference between the two groups with respect to other balance parameters $(p>0.05)$. 
Table 2

Parameters during left one-leg stance balance between normal adults and HV adults

\begin{tabular}{|c|c|c|c|c|}
\hline Parameters & $\begin{array}{l}\text { Normal adults } \\
(n=20)\end{array}$ & $\begin{array}{l}\mathrm{HV}^{\mathrm{a}} \text { Adults } \\
(\mathrm{n}=20)\end{array}$ & Z & $\mathbf{p}$ \\
\hline Forefoot $\mathrm{COP}^{\mathrm{b}} \mathrm{X}(\mathrm{mm})$ & $-48.58 \pm 44.20^{c}$ & $-50.75 \pm 38.54$ & -0.487 & 0.626 \\
\hline Forefoot COP Y (mm) & $59.58 \pm 31.63$ & $60.95 \pm 26.57$ & -0.703 & 0.482 \\
\hline Backfoot COP X (mm) & $-23.48 \pm 24.40$ & $-36.52 \pm 32.60$ & -1.488 & 0.137 \\
\hline Backfoot COP Y (mm) & $-44.39 \pm 13.01$ & $-48.09 \pm 12.83$ & -0.866 & 0.387 \\
\hline $\operatorname{COP} \times(\mathrm{mm})$ & $-25.17 \pm 51.41$ & $-32.46 \pm 38.30$ & -0.730 & 0.465 \\
\hline $\operatorname{COP} Y(m m)$ & $21.11 \pm 40.94$ & $12.60 \pm 35.73$ & -1.082 & 0.279 \\
\hline COP path (mm) & $922.37 \pm 203.84$ & $1235.20 \pm 622.40$ & -1.434 & 0.152 \\
\hline COP velocity (mm/sec) & $30.75 \pm 6.79$ & $41.17 \pm 20.75$ & -1.434 & 0.152 \\
\hline Length of minor axis (mm) & $24.59 \pm 4.29$ & $30.92 \pm 9.54$ & -2.137 & 0.033 \\
\hline Length of major axis (mm) & $39.22 \pm 7.49$ & $62.45 \pm 33.54$ & -2.570 & 0.010 \\
\hline Angle btw $\mathrm{Y}$ and major axis (deg) & $18.38 \pm 10.62$ & $14.84 \pm 11.55$ & -1.353 & 0.176 \\
\hline Deviation X (mm) & $-25.16 \pm 51.41$ & $-32.28 \pm 38.42$ & -0.676 & 0.499 \\
\hline Deviation Y (mm) & $21.09 \pm 40.93$ & $12.65 \pm 35.98$ & -1.082 & 0.279 \\
\hline Forefoot force (\%) & $67.13 \pm 15.54$ & $59.19 \pm 11.13$ & -2.056 & 0.040 \\
\hline Backfoot force (\%) & $31.20 \pm 13.54$ & $39.14 \pm 12.23$ & -2.110 & 0.035 \\
\hline Total force (\%) & $60.51 \pm 16.67$ & $66.57 \pm 17.19$ & -1.001 & 0.317 \\
\hline
\end{tabular}

\section{Unipedal Balance Test On The Right Foot}

The results of the unipedal balance test on the right foot in the HV and normal groups are presented in Table 3. The length of the major and minor axes significantly increased in the HV group compared with the normal group $(p<0.05)$. However, the other balance parameters were not significantly different between the groups $(p>0.05)$. 
Table 3

Parameters during right one-leg stance balance between normal adults and HV adults

\begin{tabular}{|c|c|c|c|c|}
\hline Parameters & $\begin{array}{l}\text { Normal adults } \\
(n=20)\end{array}$ & $\begin{array}{l}\text { HVa Adults } \\
(n=20)\end{array}$ & $\mathbf{Z}$ & $\mathbf{p}$ \\
\hline Forefoot $\mathrm{COP}^{\mathrm{b}} \mathrm{X}(\mathrm{mm})$ & $56.55 \pm 100.83^{c}$ & $58.25 \pm 41.56$ & -1.758 & 0.079 \\
\hline Forefoot COP Y (mm) & $47.64 \pm 21.92$ & $69.36 \pm 40.48$ & -1.812 & 0.070 \\
\hline Backfoot COP X (mm) & $49.57 \pm 100.44$ & $35.92 \pm 26.14$ & -0.947 & 0.344 \\
\hline Backfoot COP Y (mm) & $-32.44 \pm 12.52$ & $-34.18 \pm 13.17$ & -0.784 & 0.433 \\
\hline $\operatorname{COP} \times(\mathrm{mm})$ & $33.53 \pm 104.39$ & $38.58 \pm 51.68$ & -1.650 & 0.099 \\
\hline $\operatorname{COPY}(\mathrm{mm})$ & $7.62 \pm 27.06$ & $33.36 \pm 53.30$ & -1.569 & 0.117 \\
\hline COP path (mm) & $1108.09 \pm 333.01$ & $1326.58 \pm 443.75$ & -1.353 & 0.176 \\
\hline COP velocity (mm/sec) & $36.94 \pm 11.10$ & $44.22 \pm 14.79$ & -1.353 & 0.176 \\
\hline Length of minor axis (mm) & $28.38 \pm 7.47$ & $33.77 \pm 7.16$ & -2.489 & 0.013 \\
\hline Length of major axis (mm) & $43.85 \pm 12.95$ & $58.14 \pm 23.40$ & -2.110 & 0.035 \\
\hline Angle btw $Y$ and major axis (deg) & $18.38 \pm 12.45$ & $23.14 \pm 16.94$ & -0.866 & 0.387 \\
\hline Deviation X (mm) & $33.41 \pm 104.44$ & $38.47 \pm 51.68$ & -1.596 & 0.110 \\
\hline Deviation Y (mm) & $7.71 \pm 27.05$ & $33.41 \pm 53.20$ & -1.569 & 0.117 \\
\hline Forefoot force (\%) & $67.19 \pm 20.89$ & $67.92 \pm 20.61$ & -0.162 & 0.871 \\
\hline Backfoot force (\%) & $31.14 \pm 18.06$ & $28.75 \pm 14.24$ & -0.406 & 0.685 \\
\hline Total force (\%) & $49.41 \pm 20.71$ & $62.20 \pm 22.46$ & -1.867 & 0.062 \\
\hline
\end{tabular}

\section{Evaluation Of Gait Characteristics (left Foot)}

The parameters derived from the analysis of the left foot while walking are summarized by group in Table 4. The time change from the heel to forefoot, expressed in seconds and as percentage change, was significantly shorter in the HV group than in the normal group $(p<0.05)$. However, the other gait parameters were not significantly different between the groups $(p>0.05)$. 
Table 4

Parameters in left foot during gait between normal adults and HV adults

\begin{tabular}{|c|c|c|c|c|}
\hline Parameters & Normal adults & $\mathrm{HV}^{\mathrm{a}}$ Adults & Z & p \\
\hline Foot rotation (deg) & $5.16 \pm 4.53^{b}$ & $6.58 \pm 4.49$ & -1.136 & 0.256 \\
\hline Step length (cm) & $61.24 \pm 7.74$ & $59.82 \pm 3.56$ & -0.906 & 0.365 \\
\hline Stance phase (\%) & $62.83 \pm 1.92$ & $63.04 \pm 0.77$ & -0.785 & 0.433 \\
\hline Load response (\%) & $12.91 \pm 1.87$ & $12.98 \pm 1.45$ & -0.081 & 0.935 \\
\hline Mid stance (\%) & $37.00 \pm 1.69$ & $37.26 \pm 1.76$ & -0.839 & 0.402 \\
\hline Pre-swing (\%) & $12.94 \pm 1.84$ & $12.86 \pm 1.03$ & -0.257 & 0.797 \\
\hline Swing phase (\%) & $37.17 \pm 1.92$ & $36.96 \pm 0.77$ & -0.785 & 0.433 \\
\hline Step time (sec) & $0.53 \pm 0.05$ & $0.53 \pm 0.043$ & -0.108 & 0.914 \\
\hline Length of gait line (mm) & $215.63 \pm 12.95$ & $209.41 \pm 19.71$ & -0.866 & 0.387 \\
\hline Single support line (mm) & $116.94 \pm 9.48$ & $114.55 \pm 6.77$ & -1.542 & 0.123 \\
\hline Time change heel to forefoot (sec) & $0.28 \pm 0.049$ & $0.25 \pm 0.05$ & -2.016 & 0.044 \\
\hline Time change heel to forefoot (\%) & $42.48 \pm 5.62$ & $36.98 \pm 6.71$ & -3.003 & 0.003 \\
\hline Maximum force of forefoot $(\mathrm{N})$ & $700.04 \pm 131.02$ & $707.77 \pm 144.12$ & -0.162 & 0.871 \\
\hline Maximum force of midfoot $(\mathrm{N})$ & $117.48 \pm 63.49$ & $135.24 \pm 79.66$ & -0.541 & 0.588 \\
\hline Maximum force of heel $(\mathrm{N})$ & $487.18 \pm 72.07$ & $481.65 \pm 82.66$ & -0.298 & 0.766 \\
\hline Maximum pressure of forefoot $(\mathrm{N} / \mathbb{\mathbb { }})$ & $50.94 \pm 9.70$ & $46.04 \pm 8.32$ & -1.082 & 0.279 \\
\hline Maximum pressure of midfoot $(\mathrm{N} / \mathbb{\mathbb { }})$ & $17.66 \pm 9.87$ & $17.46 \pm 6.38$ & -0.433 & 0.665 \\
\hline Maximum pressure of Heel $\left(\mathrm{N} / \mathrm{cm}^{2}\right)$ & $39.78 \pm 7.05$ & $38.29 \pm 9.44$ & -1.163 & 0.245 \\
\hline
\end{tabular}

\section{Evaluation Of Gait Characteristics (right Foot)}

The parameters derived from the analysis of the right foot while walking are summarized by group in Table 5. The time change from the heel to forefoot, expressed as percentage change, was significantly shorter in the HV group than in the normal group $(p<0.05)$. However, the other gait parameters were not significantly different between the groups $(p>0.05)$. 
Table 5

Parameters in the right foot during gait between normal adults and HV adults

\begin{tabular}{|c|c|c|c|c|}
\hline Parameters & Normal adults & HV ${ }^{a}$ Adults & $\mathbf{Z}$ & $\mathbf{p}$ \\
\hline Foot rotation (deg) & $7.56 \pm 4.92^{b}$ & $8.15 \pm 5.31$ & -0.676 & 0.499 \\
\hline Step length (cm) & $61.79 \pm 6.56$ & $59.52 \pm 4.98$ & -1.515 & 0.130 \\
\hline Stance phase (\%) & $62.87 \pm 1.44$ & $61.96 \pm 5.09$ & -0.325 & 0.745 \\
\hline Load response (\%) & $12.79 \pm 1.71$ & $13.03 \pm 1.22$ & -0.839 & 0.402 \\
\hline Mid stance (\%) & $37.42 \pm 1.93$ & $36.84 \pm 1.23$ & -1.353 & 0.176 \\
\hline Pre-swing (\%) & $12.65 \pm 1.68$ & $12.94 \pm 2.06$ & -0.298 & 0.766 \\
\hline Swing phase (\%) & $37.13 \pm 1.44$ & $36.39 \pm 3.04$ & -0.216 & 0.829 \\
\hline Step time (sec) & $0.52 \pm 0.05$ & $0.54 \pm 0.05$ & -1.233 & 0.218 \\
\hline Length of gait line (mm) & $212.99 \pm 15.32$ & $208.52 \pm 17.87$ & -0.622 & 0.534 \\
\hline Single support line $(\mathrm{mm})$ & $115.80 \pm 10.55$ & $112.29 \pm 8.25$ & -1.272 & 0.204 \\
\hline Time change heel to forefoot (sec) & $0.26 \pm 0.05$ & $0.24 \pm 0.05$ & -1.678 & 0.093 \\
\hline Time change heel to forefoot (\%) & $40.03 \pm 7.27$ & $35.55 \pm 7.76$ & -2.191 & 0.028 \\
\hline Maximum force of forefoot $(\mathrm{N})$ & $708.98 \pm 123.06$ & $719.71 \pm 149.87$ & -0.081 & 0.935 \\
\hline Maximum force of midfoot $(\mathrm{N})$ & $117.03 \pm 78.21$ & $143.63 \pm 92.73$ & -1.245 & 0.213 \\
\hline Maximum force of Heel $(\mathrm{N})$ & $473.90 \pm 73.59$ & $478.44 \pm 73.88$ & -0.460 & 0.646 \\
\hline Maximum pressure of forefoot $\left(\mathrm{N} / \mathrm{cm}^{2}\right)$ & $48.78 \pm 7.32$ & $46.84 \pm 9.90$ & -0.730 & 0.465 \\
\hline Maximum pressure of midfoot $\left(\mathrm{N} / \mathrm{cm}^{2}\right)$ & $15.04 \pm 7.06$ & $16.84 \pm 5.68$ & -1.380 & 0.168 \\
\hline Maximum pressure of Heel $(\mathrm{N} / \mathbb{\nabla})$ & $39.47 \pm 10.30$ & $38.22 \pm 11.07$ & -0.676 & 0.499 \\
\hline
\end{tabular}

\section{Simple Linear Regression Analysis}

A simple linear regression analysis was performed to determine the relationship of HVA with balance and gait parameters, and the results are summarized in Tables 6 (left foot) and 7 (right foot). In the linear regression models derived for gait parameters, the time change from the heel to forefoot, expressed in seconds and as percentage change, was significant in the left foot $(p<0.05)$. However, other left gait parameters did not show significant differences $(p<0.05)$. In the assessment of standing balance on the left foot, a statistically significant regression equation was derived for the major and minor axes $(p<$ 
0.05). The time change from the heel to forefoot, expressed as percent change, was also significant ( $p<$ $0.05)$ in the right foot. However, other gait parameters did not show significant differences $(p>0.05)$. Additionally, other balance parameters measured while standing on the right foot $(p>0.05)$ were not statistically significantly different $(p>0.05)$.

Table 6

Equations for $\mathrm{HV}$ angles of the left foot on the balance and gait parameters by simple regression analysis

\begin{tabular}{|lllllll|}
\hline Parameters & Regression equation & $\mathbf{r}$ & $\mathbf{R}^{2}$ & $\boldsymbol{\beta}$ & $\mathbf{F}$ & $\mathbf{p}$ \\
\hline $\begin{array}{l}\text { Time to change heel to } \\
\text { forefoot (sec) }\end{array}$ & $\begin{array}{l}0.302+(-0.002 \times \mathrm{HV} \\
\text { angle) }\end{array}$ & 0.336 & 0.113 & -0.336 & 4.841 & 0.034 \\
\hline $\begin{array}{l}\text { Time to change heel to } \\
\text { forefoot (\%) }\end{array}$ & $\begin{array}{l}45.622+(-0.339 \times \mathrm{HV} \\
\text { angle) }\end{array}$ & 0.386 & 0.149 & -0.386 & 6.667 & 0.014 \\
\hline Length of minor axis & $\begin{array}{l}20.374+(0.424 \times \mathrm{HV} \\
\text { angle) }\end{array}$ & 0.408 & 0.166 & 0.408 & 7.579 & 0.009 \\
\hline Length of major axis & $\begin{array}{l}29.595+(1.221 \times \mathrm{HV} \\
\text { angle) }\end{array}$ & 0.350 & .0123 & 0.350 & 5.310 & 0.027 \\
\hline aHalux valgus & & & & & & \\
\hline
\end{tabular}

Table 7

Equations for $\mathrm{HV}$ angles of the right foot on the balance and gait parameters by simple regression analysis

\begin{tabular}{|lllllll|}
\hline Parameters & Regression equation & $\mathbf{r}$ & $\mathbf{R}^{2}$ & $\boldsymbol{\beta}$ & $\mathbf{F}$ & $\mathbf{p}$ \\
\hline $\begin{array}{l}\text { Time to change heel to forefoot } \\
(\%)\end{array}$ & $\begin{array}{l}43.421+(-0.349 \times \\
\left.\mathrm{HV}^{\mathrm{a}}\right)\end{array}$ & 0.329 & 0.108 & -0.329 & 4.614 & 0.038 \\
\hline Length of minor axis & $\begin{array}{l}24.161+(0.428 \times \\
\mathrm{HV})\end{array}$ & 0.406 & 0.165 & 0.406 & 7.509 & 0.009 \\
\hline aHalux valgus & & & & & & \\
\hline
\end{tabular}

\section{Discussion}

In this study, data were not distinguished by group in the simple linear regression analysis. In the assessment of one-leg stance balance, the length of the major and minor axes significantly increased in the HV group compared with the normal group. Among gait parameters, time change from the heel to forefoot, expressed as percentage change, was significantly shorter in the HV group than in the normal group. In linear regression analysis using HVA as an independent variable, the minor axis and time changes from heel to forefoot, expressed as percentage change, were significantly different between the groups. However, other balance and gait parameters were not significantly different between the groups. 
A comparison of the two groups revealed no significant differences in most balance and gait parameters evaluated. This finding may be attributable to the young age of subjects (i.e., in their $20 \mathrm{~s}$ ) and their health status, as they did not complain of pain and other symptoms, according to the inclusion criteria. Nonetheless, a few balance and gait parameters were significantly different between the groups.

In the comparison of parameters used to assess one-leg stance balance, the length of the major and minor axes was significantly longer in the right and left feet in the HA group than in the normal group. During standing on the left leg, force (\%) on the forefoot was significantly lower in the HA than in the normal group, whereas force (\%) on the back foot was significantly higher in the HA group than in the normal group. According to Cavanagh et al. [23], the plantar pressure distribution is located in the rear foot $(60 \%)$, midfoot ( $8 \%)$, forefoot region ( $28 \%$ ), and toes (about $4 \%$ ), and the forefoot's peak pressure occurs under the second metatarsal head. Nyska et al. [24] stated that HV altered typical biomechanics, caused functional impairment of the great toe, and increased central metatarsal loading. Hutton and Dhanendran [25] suggested that changes in HV feet were accompanied with reduced weight-bearing function of the great toe, and reported increased peak pressure under the medial metatarsal head and load imposed on the central forefoot region as a typical pattern. In addition, Bryant et al. [26] reported that the peak pressure increased under the great toe region. However, Thomas and Barrington [6] claimed that the contact area of the great toe could be reduced due to the deformity and infection of the first metatarsal. Unlike results of previous studies, we found a significantly higher force distribution (\%) in the back foot and lower force distribution (\%) in the forefoot. This discrepancy can be explained by the young age of our subjects. However, this finding suggests the possibility that HV can cause distribution changes in plantar pressure; that is, toe deformity is closely associated with plantar pressure [19]. In contrast, this study found that HVA can help increase the path of the COP shift rather than plantar pressure, according to linear regression analysis. Carvalho et al. [27] asserted that $\mathrm{HV}$ could affect sway during shifts of the COP to varying degrees, depending on the forefoot width and foot arch. Wen et al. [13] reported that contact time extends as motions increase loading on the lateral and medial rear foot regions. Although our study found different distribution patterns of plantar pressure in the HV feet compared with that reported in previous studies, such a difference implies that pressure could increase in some parts of the plantar relative to other parts of the plantar. As a result, COP bearing capability and shift distance may be affected more frequently in HV.

In the comparison of gait parameters between the two groups, time change from the heel to forefoot, expressed as percentage change, was significantly shorter in the right and left feet in the HA group than in the normal group. In the assessment of left foot-related parameters, time change from the heel to forefoot, expressed as elapsed time, was also significantly shorter in the HA group than in the normal group. Canseco et al. [28] reported that foot-related disorders affect gait parameters. Nix et al. [29] reported that the experimental group with $\mathrm{HA}$ had changes in the weight-bearing pattern under the great toe and metatarsal head while walking. Menz and Morris [4] reported decreases in gait speed and phases in the HA group compared with the normal group. Deschamps et al. [1] stated that patients with HA demonstrated similar gait pattern and time to those of healthy individuals during the entire stance and swing periods and that there was a difference in plantar pressure distribution during walking between the 
groups. According to Wen et al. [13], the tendency of increasing weight bearing at the midfoot, once started in the early stance phase of gait, eventually delays weight transfer from the medial side to lateral side of the foot. In the present study, we found no differences in the entire stance period as expressed in seconds and as a percentage. However, time change from the heel to forefoot was shorter, which indicates a shorter duration of weight bearing at the forefoot. When walking is repeated with this parameter, the forefoot's pressure will aggregate, increasing the risk of cumulative damage to the foot.

The plantar pressure measurement serves as an indicator of foot and ankle functions during walking and other functional activities. The size and pattern of pressure distribution at the plantar surface depends on the structural and functional status of the foot and ground state. Abnormal foot pressures are associated with not only pain but foot deformity [30].

This study has some limitations. Pressure distribution parameters were used in the gait analysis of the forefoot as a whole rather than the hallux region; thus, further analysis of the forefoot segments is required in future studies. Given that only young subjects in their $20 \mathrm{~s}$ participated in this study, a larger study sample that includes older adults is necessary in further studies.

\section{Conclusions}

The present study identified the relationship of HVA with balance and gait performance in young adults by measuring the HVA and analyzing static and dynamic parameters obtained from one-leg stance and a 6-m walking test. Given that walking is a repetitive activity, young adults with HV could potentially develop disease or cumulative damage to the feet in later stages of life. Future studies are needed to address this possibility through accurate measurements and analysis.

\section{List Of Abbreviations}

COP: center of pressure

$\mathrm{HV}$ : Hallux valgus

HVA: Hallux valgus angle

\section{Declarations}

Ethics approval and consent to participate

The study protocol was approved by the institutional review board of Jeonju University (no. jjIRB-180713HR-2018-0708). And also, written informed consent form was received from each participant.

Consent for publication

Not applicable 
Availability of data and material

All data generated or analysed during this study are included in this published article.

Competing interests

The authors declare that they have no competing interests.

Funding

Not applicable

Authors' contributions

Ji, Park, Lee, Yoo, Ko, and Woo were involved in study conception and design. Ji, Park,

Lee, and Yoo were involved data collection, and Woo was involved data analysis and interpretation. All authors were involved drafting the manuscript. All authors have read and approved the final manuscript.

Acknowledgements

Not applicable

Authors' information (optional)

\section{References}

1. Deschamps K, Birch I, Desloovere K, Matricali GA. The impact of hallux valgus on foot kinematics: a cross-sectional, comparative study. Gait Posture. 2010;32(1):102-6.

2. Glasoe WM, Nuckley DJ, Ludewig PM. Hallux valgus and the first metatarsal arch segment: a theoretical biomechanical perspective. Phys Ther. 2010;90(1):110-20.

3. Roddy E, Zhang W, Doherty M. Prevalence and associations of hallux valgus in a primary care population. Arthritis Rheum. 2008;59(6):857-62.

4. Menz HB, Morris ME. Footwear characteristics and foot problems in older people. Gerontology. 2005;51:346-51.

5. Nguyen US, Hillstrom HJ, Li W, Dufour AB, Kiel DP, Procter-Gray E, et al. Factors associated with hallux valgus in a population-based study of older women and men: the MOBILIZE Boston Study. Osteoarthritis Cartilage. 2010;18(1):41-6.

6. Thomas S, Barrington R. Hallux valgus. Orthopaedics and Trauma. 2003;17(4):299-307.

7. Lin JS, Bustillo J. Surgical treatment of hallux valgus: a review. Current Opinion in Orthopaedics. 2007;18(2):112-7.

8. Mann RA, Coughlin MJ. Adult hallux valgus. In: Coughlin MJ, Mann RA. Surgery of the foot and ankle. 7th ed. 7St. Louis: Mosby Inc; 1999. P 150. 
9. Pique-Vidal C, Vila J. A geometric analysis of hallux valgus: correlation with clinical assessment of severity. J Foot Ankle Res. 2009;2:15.

10. Stewart S, Ellis R, Heath M, Rome K. Ultrasonic evaluation of the abductor halluces muscle in hallux valgus: a cross-sectional observational study. BMC Musculoskeletal Disord. 2013;14:45.

11. Suzuki J, Tanaka Y, Takaoka T, Kadono K, Takakura Y. Axial radiographic evaluation in hallux valgus: evaluation of the transverse arch in the forefoot. J Orthop Sci. 2004;9(5):446-51.

12. Steinberg N, Finestone A, Noff M, Zeev A, Dar G. Relationship between lower extremity alignment and hallux valgus in women. Foot Ankle Int. 2013;34(6):824-31.

13. Wen J, Ding Q, Yu Z, Sun W, Wang Q, Wei K. Adaptive changes of foot pressure in hallux valgus patients. Gait Posture. 2012;36(3):344-9.

14. Nishimura A, Ito N, Nakazora S, Kato K, Ogura T, Sudo A. Does hallux valgus impair physical function? BMC Musculoskelet Disord. 2018;19(1):174.

15. Ferrari J, Hopkinson DA, Linney AD. Size and shape differences between male and female foot bones: is the female foot predisposed to hallux abducto valgus deformity? J Am Podiatr Med Assoc. 2004;94(5):434-52.

16. Schuh R, Hofstaetter SG, Adams SB Jr, Pichler F, Kristen KH, Tmka HJ. Rehabilitation after hallux valgus surgery: importance of physical therapy to restore weight bearing of the first ray during the stance phase. Phys Ther. 2009;89(9):934-45.

17. Martinez-Nova A., Sanchez-Rodriguez R, Perez-Soriano P, Llana-Belloch S, Leal-Muro A, PedreraZamorano JD. Plantar pressures determinants in mild hallux valgus. Gait Posture. 2010;32(3):425-7.

18. Bryant A. Tinley P. Singer K. Radiographic measurements and plantar pressure distribution in normal, hallux valgus and hallux limitus feet. The Foot. 2000;10(1):18-22.

19. Mickle KJ, Munro BJ, Lord SR, Menz HB, Steele JR. Gait, balance and plantar pressures in older people with toe deformities. Gait Posture. 2011;34(3):347-51.

20. Hurn SE, Vicenzino B, Smith MD. Functional impairments characterizing mild, moderate, and severe hallux valgus. Arthritis Care Res (Hoboken). 2015;67(1):80-8.

21. Moon JH, Lee HS, Jung KI, Park JH, Lee DS. Foot pressure distribution in normal subjects and patients with hallux valgus and pes planus. Journal of the Korean Academy of Rehabilitation Medicine. 1996;20(3):33.

22. Srivastava S, Chockalingam N, El Fakhri T. Radiographic angles in hallux valgus: comparison between manual and computer-assisted measurements. J Foot Ankle Surg. 2010;49(6):523-8.

23. Cavanagh PR, Morag E, Boulton AJ, Young MJ, Deffner KT, Pammer SE. The relationship of static foot structure to dynamic foot function. J Biomech. 1997;30(3):243-50.

24. Nyska M, Liberson A, McCabe $C$, Linge $K$, Klenerman L. Plantar foot pressure distribution in patients with hallux valgus treated by distal soft tissue procedure and proximal metatarsal osteotomy. Foot and Ankle Surg. 1998;4(1):35-41. 
25. Hutton W, Dhanendran $M$. The mechanics of normal and hallux valgus feet-a quantitative study. Clin Orthop Relat Res. 1981;157:7-13.

26. Bryant $A$, Tinley $P$, Singer K. Plantar pressure distribution in normal, hallux valgus and hallux limitus feet. The Foot. 1999;9(3):115-9.

27. Carvalho CE, da Silva RA, Gil AW, Oliveira MR, Nascimento JA, Pires-Oliveira DA. Relationship between foot posture measurements and force platform parameters during two balance tasks in older and younger subjects. J Phys Ther Sci. 2015;27(3):705-710.

28. Canseco K, Long J, Marks R, Khazzam M, Harris G. Quantitative characterization of gait kinematics in patients with hallux rigidus using the Milwaukee foot model. J Orthop Res. 2008;26(4):419-27.

29. Nix SE, Vicenzino BT, Collins NJ, Smith MD. Gait parameters associated with hallux valgus: a systematic review. J Foot Ankle Res. 2013;6(1):9.

30. Orlin MN, McPoil TG. Plantar pressure assessment. Phys Ther. 2000;80(4):399-409. 